\title{
Dilleniaceae endémicas del Perú
}

\author{
Blanca León ${ }^{1,2}$
}

${ }^{1}$ Museo de Historia Natural, Av. Arenales 1256, Aptdo. 14-0434, Lima 14, Perú

2 Plant Resources Center, University of Texas at Austin, Austin TX 78712 EE.UU.

blanca.leon@mail.utexas.edu

\section{Resumen}

La familia Dilleniaceae es una adición reciente a la flora endémica peruana; los endemismos han sido recolectados y reconocidos hacia fines de los 1990. Está representada en el Perú por seis géneros y 23 especies (Brako \& Zarucchi, 1993; Ulloa Ulloa et al., 2004), la mayoría lianas. En este trabajo reconocemos tres endemismos en dos géneros. Las especies endémicas ocupan la región Bosques Húmedos Amazónicos, entre los 100 y 125 m de altitud. No se encuentra representada en el Sistema Nacional de Áreas Naturales Protegidas por el Estado.

Palabras claves: Dilleniaceae, Perú, endemismo, plantas endémicas.

\section{Abstract}

This family is a recent addition to the endemic Peruvian flora; its endemic taxa were recognized in the late 1990s. The Dilleniaceae are represented in Peru by six genera and 23 species (Brako \& Zarucchi, 1993; Ulloa Ulloa et al., 2004), mostly lianas. Here we recognize three endemic species in two genera. Endemic Dilleniaceae species are found in the Humid Lowland Amazonian Forests region between 100 and 125 m elevation. None of the species has been recorded to date in Peru's protected area system.

Keywords: Dilleniaceae, Peru, endemism, endemic plants.

\section{Doliocarpus gentryi G. Aymard \& Miller}

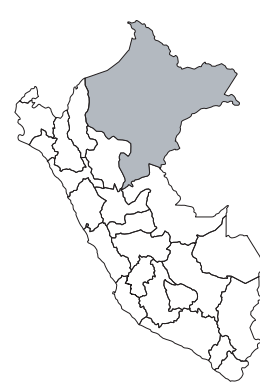

\section{EN, B1a}

Publicación: Candollea 49(1): 176, f. 1. 1994.

Colección tipo: A.H. Gentry et al. 61793

Herbarios: MO; AMAZ, USM!.

Nombre común: Puca huasca.

Registro departamental: LO.

Regiones Ecológicas: BHA; $130 \mathrm{~m}$.

SINANPE: Sin registro.

Herbarios peruanos: AMAZ (isotipo citado), USM (isotipo).

Observaciones: Liana conocida de unas pocas localidades en la Provincia de Maynas, entre ella un área de protección privada (Explorama). Vásquez (1997) mencionó que ocupa ambientes en bosque no intervenido.

\section{Doliocarpus pipolyi G. Aymard}

\section{EN, B1ab(iii)}

Publicación: Novon 3(4): 318. 1993. Colección tipo: J. Pipoly et al. 13420 Herbarios: MO, PORT; AMAZ, USM!. Nombre común: Puca huasca. Registro departamental: LO. Regiones Ecológicas: BHA; 100-123 m. SINANPE: Sin registro.

Herbarios peruanos: AMAZ (isotipo citado), USM (isotipo).

Observaciones: Liana conocida de la cuenca del Napo. Una población está representada en una reserva privada (Explorama Lodge). Esta especie es propia de bosques no inundables. Fuera de la reserva la amenaza principal a esta especie proviene de la destrucción de hábitat.

\section{Neodillenia peruviana G. Aymard}

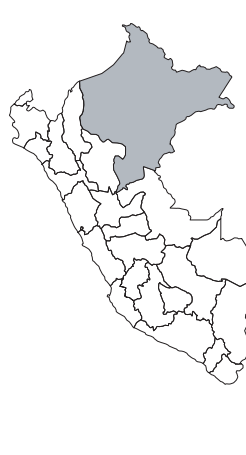
EN, B1a

Publicación: Harvard Pap. Bot. 10: 126. 1997.

Colección tipo: R. Vásquez \& N. Jaramillo 13057

Herbarios: MO; AMAZ, USM!.

Nombre común: Desconocido.

Registro departamental: LO.

Regiones Ecológicas: BHA; 116-130 m. SINANPE: Sin registro.

Herbarios peruanos: AMAZ (isotipo citado+1), USM (isotipo).

Observaciones: Esta especie fue descrita de una planta recolectada en una subcuenca del Amazonas; sus poblaciones se hallan en un área de protección privada (Explornapo). Por su hábito, esta especie es dependiente de bosques, por lo que la amenaza principal es la deforestación. 